\title{
Graphene - A Promising Electrode Material in Liquid Cell Electrochemistry
}

Shu Fen Tan ${ }^{1}$, Kate Reidy ${ }^{2}$, Serin Lee ${ }^{3}$, Julian Klein ${ }^{4}$, Nicholas Schneider ${ }^{5}$, Hae Yeon Lee ${ }^{6}$ and Frances Ross 4

${ }^{1}$ MIT, Massachusetts, United States, ${ }^{2}$ Massachusetts Institute of Technology (MIT), United States, ${ }^{3}$ MIT, Cambridge, Massachusetts, United States, ${ }^{4}$ MIT, United States, ${ }^{5}$ Renata Global, United States, ${ }^{6}$ Massachusetts Institute of Technology, United States

Two-dimensional (2D) materials such as graphene and transition metal dichalcogenides (TMDs) are potential candidate materials for electrodes due to their exceptional intrinsic properties, particularly high specific surface area, electrical charge mobility, thermal conductivity and mechanical strength. ${ }^{1}$ These materials have been explored as next generation electrode materials for electrical and optical devices, ${ }^{2}$ supercapacitors, ${ }^{3}$ organic electronics ${ }^{4}$ and dye-sensitized solar cells. ${ }^{5}$ In contrast, for electrochemical liquid cell TEM, the most common electrode materials are $\mathrm{Pt}^{6,7}$ and $\mathrm{Au}^{8,9}$ in most of the workflows that have been used in fabricating state-of-the-art electrochemical MEMS thin window cells. Pt and Au are undoubtedly beneficial due to their stability to corrosion and the high conductivity of even thin films. However, their large mass contrast contributes a non-uniform background due to the polycrystalline nature of the films. An ideal electrode material in liquid cell TEM electrochemistry should not scatter electrons strongly because the resolution in liquid cell TEM is often dose-limited due to the sensitivity of the electrolyte to electron beam irradiation. ${ }^{10}$ The signal to noise ratio, which controls resolution in these circumstances, depends on the mass thickness of all the components in the electrochemical cell. This includes the windows and liquid layer but also the electrode, particularly when observing phenomena that take place over the surface of the electrode rather than only around the edges. In addition, the electrode material must also ensure consistent and reliable electrochemical measurements so as to allow the process that takes place within the liquid cell to replicate the "real life" process as closely as possible.

Here, we focus on the opportunities arising from the use of graphene and other 2D materials in liquid cell electrochemistry. To establish whether 2D materials can indeed evolve into a reliable electrode material for both electroanalytical chemistry and electron microscopy, we first develop a reproducible protocol in transferring 2D materials onto microfabricated liquid cell chips to achieve reliable electrical contact and mechanical robustness (Figure 1). Next, we examine the suitability of 2D materials as promising electrode materials, considering different aspects such as suitability for electrodeposition, wettability, stability in terms of mechanical, electrical and beam damage, as well as the resolution improvement. We examine a well-characterized system, $\mathrm{Cu}$ nucleation and growth from acidic solution under potentiostatic conditions (Figure 2), to compare the process on graphene with the similar process on other metal electrodes. It is interesting to note that the island growths are different in each potential pulse and we further investigate the reproducibility of these metal nucleation events on other 2D materials. Our real-time imaging shows that electrochemical processes on 2D materials are readily visible and intrinsically fascinating. Exploring the possibility of 2D materials as electrode materials potentially opens up new opportunities for investigating a wide range of problems pertaining to battery materials reactions and electrocatalysis. ${ }^{11}$ 


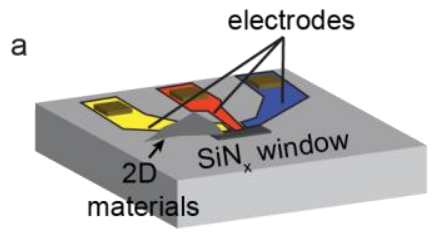

b

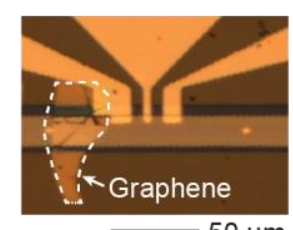

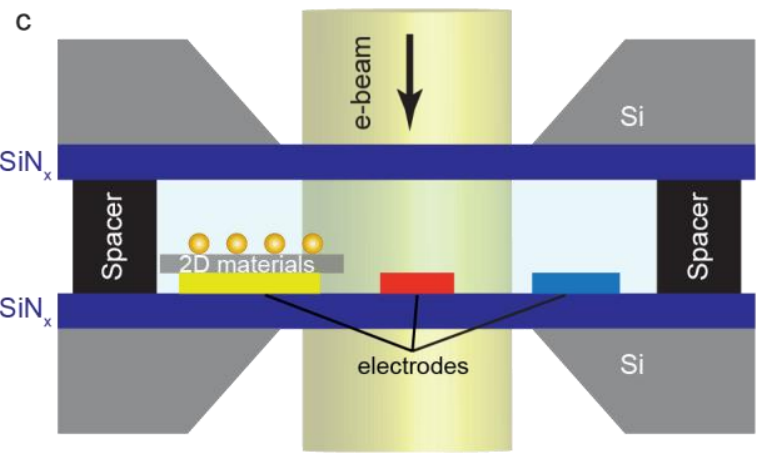

Figure 1. Transfer mechanism of exfoliated 2D materials onto electrochemical liquid cell chip. (a) Schematic of transferred 2D materials onto electrochemical liquid cell chip. (b) Optical image showing the placement of 2D materials, here graphene onto one of the Pt electrodes on an electrochemical liquid cell chip as working electrode, unmodified $\mathrm{Pt}$ electrodes act as quasi-reference and counter electrodes respectively. (c) Schematic of electrochemical liquid cell experiment set-up.
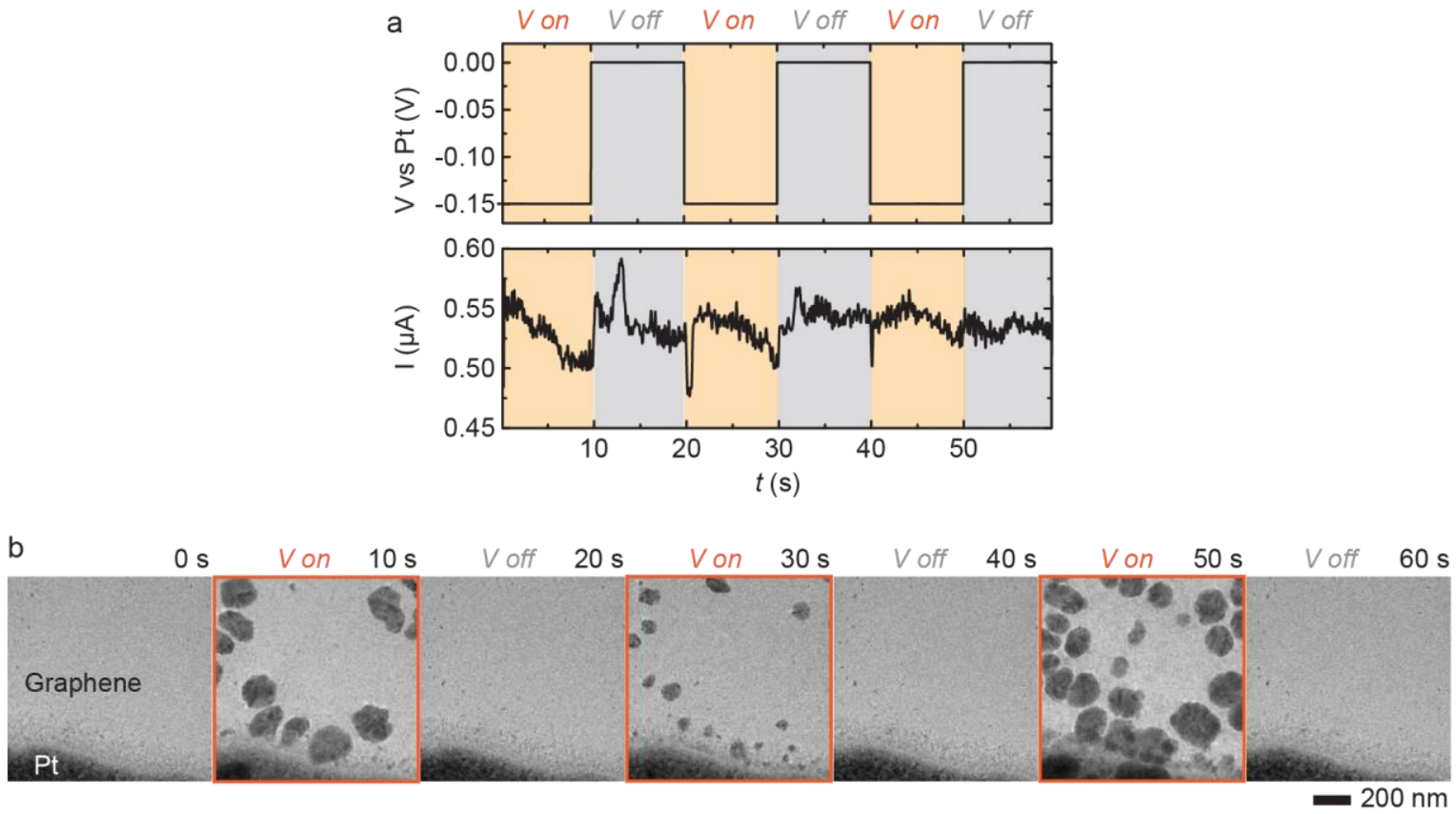

Figure 2. Pulse voltammetry of $\mathrm{Cu}$ nanoparticle (NP) deposition and dissolution under electrochemical control. (a) Driving potential (-150 mV in 10-s pulses, followed by $0 \mathrm{mV}$ in 10 -s pulses for three cycles), the resulting current responses and (b) Time-lapsed TEM sequential images obtained in an electrochemical liquid cell filled with $0.1 \mathrm{M} \mathrm{CuSO} 4+0.1 \mathrm{M} \mathrm{H} 2 \mathrm{SO} 4$ electrolyte solution.

\section{References}

1. Hynek, D. J.; Pondick, J. V.; Cha, J. J., The development of 2D materials for electrochemical energy applications: A mechanistic approach. APL Materials 2019, 7 (3), 030902. 
2. Jo, G.; Choe, M.; Lee, S.; Park, W.; Kahng, Y. H.; Lee, T., The application of graphene as electrodes in electrical and optical devices. Nanotechnology 2012, 23 (11), 112001.

3. Ke, Q.; Wang, J., Graphene-based materials for supercapacitor electrodes - A review. Journal of Materiomics 2016, 2 (1), 37-54.

4. $\quad$ Pang, S.; Hernandez, Y.; Feng, X.; Müllen, K., Graphene as Transparent Electrode Material for Organic Electronics. Adv. Mater. 2011, 23 (25), 2779-2795.

5. Wang, H.; Hu, Y. H., Graphene as a counter electrode material for dye-sensitized solar cells. Energy Environ. Sci. 2012, 5 (8), 8182-8188.

6. Hodnik, N.; Dehm, G.; Mayrhofer, K. J. J., Importance and Challenges of Electrochemical in Situ Liquid Cell Electron Microscopy for Energy Conversion Research. Acc. Chem. Res. 2016, 49 (9), 2015-2022.

7. Schneider, N. M.; Park, J. H.; Grogan, J. M.; Steingart, D. A.; Bau, H. H.; Ross, F. M., Nanoscale evolution of interface morphology during electrodeposition. Nature Communications 2017, 8 (1), 2174.

8. Williamson, M. J.; Tromp, R. M.; Vereecken, P. M.; Hull, R.; Ross, F. M., Dynamic microscopy of nanoscale cluster growth at the solid-liquid interface. Nat Mater 2003, 2 (8), 532-536.

9. Radisic, A.; Vereecken, P. M.; Hannon, J. B.; Searson, P. C.; Ross, F. M., Quantifying Electrochemical Nucleation and Growth of Nanoscale Clusters Using Real-Time Kinetic Data. Nano Lett. 2006, 6 (2), 238-242.

10. de Jonge, N.; Houben, L.; Dunin-Borkowski, R. E.; Ross, F. M., Resolution and aberration correction in liquid cell transmission electron microscopy. Nature Reviews Materials 2019, 4 (1), 61-78.

11. This work made use of facilities and instrumentation supported by NSF through the Massachusetts Institute of Technology Materials Research Science and Engineering Center under Grant DMR-1419807. 